\title{
RELAÇÃO ENTRE VARIÁVEIS ASSOCIADAS AO AMBIENTE DE FORMAÇÃO DAS LINHAS DE INSTABILIDADE E EVENTOS DE GRANDE ESCALA
}

\section{ARTIGO ORIGINAL}

CARNEIRO, Izana Oliveira ${ }^{1}$

OLIVEIRA, Gabrielle Bezerra ${ }^{2}$

SILVA, Aline Regina dos Santos ${ }^{3}$

CARNEIRO, Izana Oliveira. OLIVEIRA, Gabrielle Bezerra. SILVA, Aline Regina dos Santos. Relação entre variáveis associadas ao ambiente de formação das linhas de instabilidade e eventos de grande escala. Revista Científica Multidisciplinar Núcleo do Conhecimento. Ano 05, Ed. 09, Vol. 08, pp. 14-24. Setembro de 2020. ISSN: 2448-0959, Link de

acesso: https://www.nucleodoconhecimento.com.br/meteorologia/linhas-deinstabilidade

\section{RESUMO}

Objetivo: Analisar a relação entre umidade específica, vento, configuração em grande escala com ênfase no El Niño Oscilação Sul (ENOS) e a formação e propagação das linhas de instabilidade (LI) amazônicas. Método: Averigua-se e compara-se dois anos de dados (2009 e 2013) para avaliar o comportamento das referidas variáveis sob influência de eventos ENOS no ambiente de formação das LI. São utilizados os softwares Climate Data Operators (CDO) e Grid Analysis and Display System (GrADS)

\footnotetext{
${ }^{1}$ Mestrado em andamento em Meteorologia. Graduação em Meteorologia.

2 Doutorado em andamento em Meteorologia. Mestrado em Meteorologia. Graduação em andamento em Física. Graduação em Meteorologia.

${ }^{3}$ Graduação em Química Industrial. Graduação em andamento em Química.
} 
para manipulação dos dados de reanálises do European Centre for Medium-Range Weather Forecasts (ECMWF). Resultados: Nota-se que o ano de 2009, em que há atuação de El Niño, apresenta menor frequência de LI, enquanto o ano de 2013, com situação de neutralidade em relação ao ENOS, apresenta maior número de LI. As diferenças positivas do teor de umidade entre a média anual dos dias com LI e sem LI, bem como a diferença da intensidade e direção dos ventos que sopram do oceano para a costa, confirmam a relevância dessas variáveis para a formação e propagação da LI amazônicas. Além da relação com o ENOS, também é considerada possível influência da ASAS e da ASAN. Tais informações tornam-se necessárias e as interações aqui consideradas, bem como outras interações devem continuar sendo estudadas, devido ao elevado impacto desses sistemas na região amazônica e norte do Nordeste do Brasil, assim como a dificuldade de prognosticá-los em razão da sua complexidade.

Palavras-chave: Amazônia, reanálises, linhas de instabilidade, umidade específica, ENOS.

\section{INTRODUÇÃO}

As linhas de instabilidade (LI) são um dos sistemas atmosféricos mais importantes e frequentes na Amazônia e norte do Nordeste do Brasil (NEB). Podem ser entendidas como conjuntos organizados de cumulonimbus, com deslocamento simultâneo, responsáveis por índices elevados de precipitação nessas regiões. Segundo afirmam Cavalcanti et al. (2009), sua causa se relaciona com a circulação da brisa marítima e com a oscilação diurna e são importantes para o transporte de calor para a alta troposfera. Sua atividade máxima pode ser observada ao anoitecer. Alcântara (2010) explica que as LI apresentam uma extensão de 1000 a $2000 \mathrm{~km}$, mas podem se estender por até $3500 \mathrm{~km}$, e propagam-se com velocidade entre 50 e $60 \mathrm{~km} / \mathrm{h}$, permanecendo no ambiente por cerca $48 \mathrm{~h}$. Durante esse período, o sistema apresenta um ciclo de vida que pode ser dividido em 6 momentos: gênesis, intensificação, maturidade, enfraquecimento, re-intensificação e dissipação, conforme explicam Garstang et al. (1994). 
Cohen et al. (1995) classificam as linhas de instabilidade de acordo com sua propagação horizontal para o interior do continente: LIC são linhas de instabilidade costeiras, que se propagam até 170 km; LIP1 são linhas de instabilidade de propagação do tipo 1, avançando entre 170 e 400 km; LIP2 são linhas de instabilidade de propagação do tipo 2, que se deslocam por mais que $400 \mathrm{~km}$ para o interior. Alcântara (2010) observa que um fator que pode estar fortemente associado com a propagação das LI são os jatos de baixos níveis (JBN). A autora verifica que a ocorrência de JBN acompanha as variações de ocorrência de linhas de instabilidade e nota-se uma tendência de jato mais intenso nas LI que se propagam do que nas que não se propagam. Sabe-se, porém, que esse fator não é o único responsável por fazer as LI se propagarem por milhares de quilômetros, sendo apenas um dos elementos que favorece a propagação (ALCÂNTARA, 2010). A partir das conclusões de Cohen et al. (1995) e Silva Dias e Ferreira (1992), percebe-se que a velocidade do vento, mais do que a direção, constitui mais um fator importante para o processo de propagação, bem como a temperatura da superfície do mar (TSM) mais elevada, facilitando a formação de ondas de leste, que podem atingir a costa norte do Brasil (ALCÂNTARA, 2010).

A região onde a linha de instabilidade amazônica se forma está compreendida entre $10^{\circ} \mathrm{N}$ e $5^{\circ} \mathrm{S}$. Cavalcanti (1982) afirma que os pontos extremos das LI acompanham o deslocamento da Zona de Convergência Intertropical (ZCIT), ficando geralmente ao sul da mesma. Quando a ZCIT está bem estabelecida, pode haver aumento no número de LI, não considerando sua propagação para o interior.

As linhas de instabilidade na Amazônia ocorrem no fim da tarde e penetram o continente, mas, durante a noite, o resfriamento radiativo provoca dissipação das nuvens, que podem ficar ativas novamente no dia posterior, à medida que a superfície aquece outra vez, como observado por Molion (1987), que explica que esse mecanismo não deve ser o único encarregado da dissipação das LI, pois em alguns casos esse processo ocorre em horários de aquecimento, sendo necessário estudar os efeitos da orografia sobre esses sistemas, assim como outros estudos, devido à complexidade do sistema convectivo em questão. 
Ainda que as linhas de instabilidade sejam um dos sistemas de mesoescala de maior relevância que atuam na Amazônia e norte do NEB, são difíceis de prognosticar e simular, em razão de suas características complexas e multiescalares (diversos processos ocorrendo em várias escalas de tempo e espaço compõem o sistema final). De tal forma que dados observados e em bases diversas são de enorme importância para uma melhor compreensão acerca da formação, intensificação, propagação e dissipação desse sistema.

Diante de tais informações e desafios a respeito do referido sistema convectivo e suas interações com diversos fatores, o presente trabalho se propõe a analisar como o ambiente de formação das linhas de instabilidade, sistema da mesoescala, pode sofrer influência da configuração em grande escala, mais precisamente de um evento de El Niño Oscilação Sul (ENOS) e de sua ausência (neutralidade) ao longo do ano, concentrando-se na análise de duas das variáveis mais relevantes para a formação das LI: umidade e vento. O trabalho de Negri et al. (2000), por exemplo, aponta para uma pronunciada redução das chuvas na região Norte do país em geral, durante os eventos de El Niño em 1992 e 1997. E aumento da precipitação em boa parte do norte da América do Sul, durante a ocorrência de La Niña em 1988 e 1989. Assim, sabendose que os eventos de ENOS influenciam fortemente a quantidade de precipitação na região amazônica, pressupõe-se que também exerçam influência sobre a frequência e intensidade das $\mathrm{LI}$, bem como sobre as variáveis envolvidas no seu ambiente de formação. Essas informações podem contribuir para a compreensão acerca das interações entre as linhas de instabilidade e outros processos sinópticos, sendo útil para elaboração de prognósticos meteorológicos e planejamento em vários setores da sociedade (comércio, agricultura, aviação, lazer, turismo, entre outros), tendo em vista os impactos que esse sistema pode causar, sejam eles positivos ou negativos.

\section{MATERIAL E MÉTODOS}

Foram utilizados, para a elaboração deste trabalho, dados de reanálises do European Centre Medium-Range Weather Forecast (ECMWF - http://ecmwf.int/datasets/) para o ano de 2009 e 2013, das variáveis umidade específica $(\mathrm{g} / \mathrm{Kg})$, componente zonal (u) do vento $(\mathrm{m} / \mathrm{s})$ e componente meridional (v) do vento $(\mathrm{m} / \mathrm{s})$, ao nível de $1000 \mathrm{hPa}$. Tais 
variáveis são algumas das que mais possuem relevância para a formação das linhas de instabilidade e por esta razão foram escolhidas para este estudo. As reanálises, que são representações atmosféricas que utilizam modelos para reconstituir a atmosfera, têm resolução de $0,75^{\circ}$ e os valores usados neste estudo correspondem

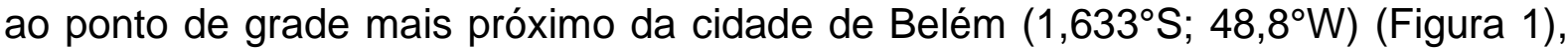
estado do Pará.

Figura 1: Localização da área de estudo. A cidade de Belém está destacada em vermelho no mapa que ocupa a área maior da figura; a miniatura no canto inferior direito destaca, em vermelho, o estado do Pará no mapa do Brasil.

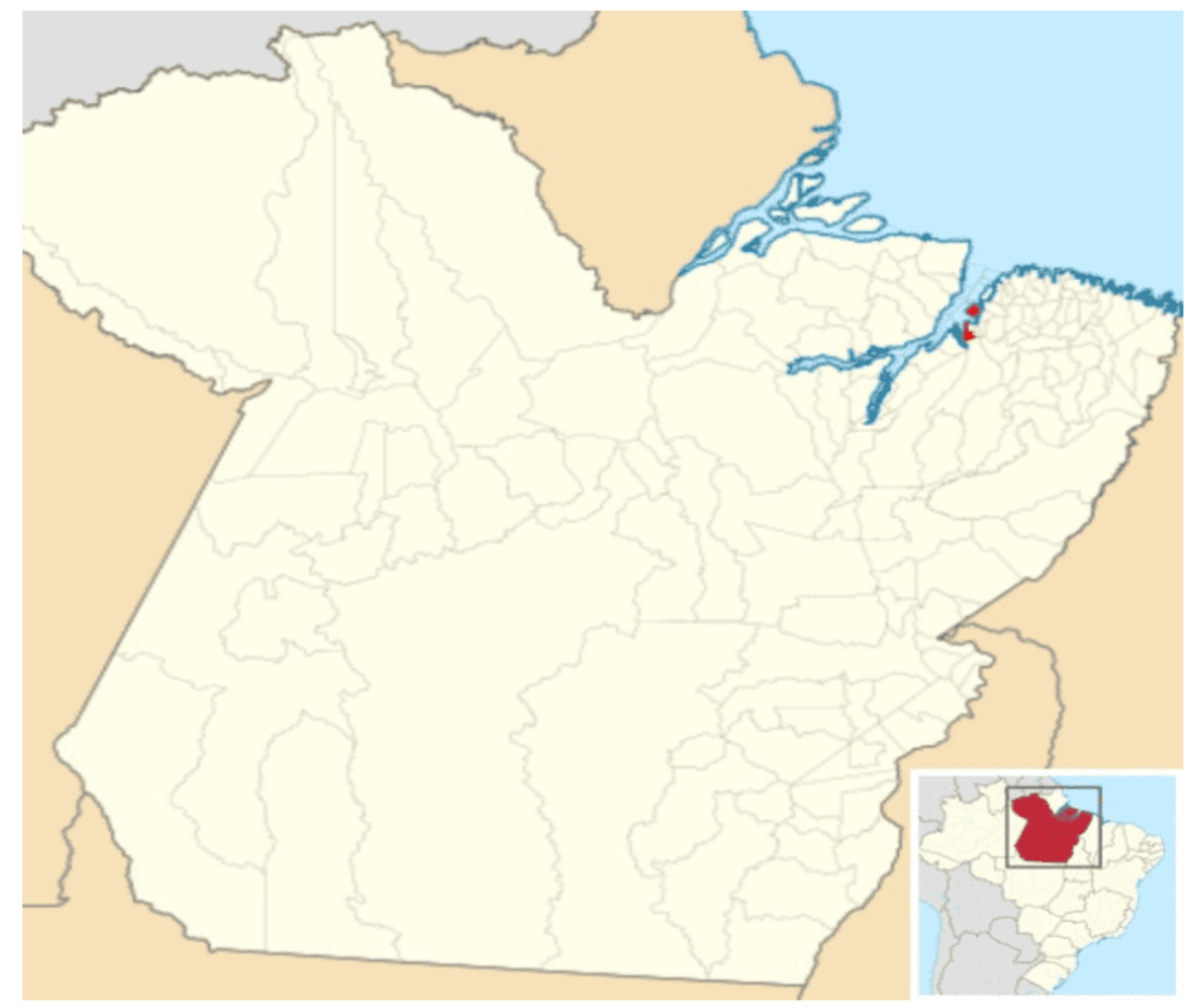

Fonte: Wikipédia (2020), adaptada pelas autoras. 
Os dados diários de ocorrência das linhas de instabilidade para os anos de 2009 e 2013 foram obtidos de forma subjetiva, baseando-se na metodologia proposta por Cohen (1989), utilizando imagens de satélite. O horário escolhido para seleção dos dados e, posteriormente, para a elaboração dos campos médios espaciais corresponde às 1200 UTC (9 horas local), horário que antecede a formação do sistema em estudo, o que facilita a observação do comportamento das variáveis analisadas. Optou-se por estudar os anos anteriormente citados, devido às particularidades que apresentam no que tange à frequência de $\mathrm{LI}$ e aos eventos de grande escala El Niño Oscilação Sul (ENOS), verificados a partir do site do Climate Prediction Center / National Centers for Environmental Prediction (CPC/NCEP) da National Oceanic and Atmosferic Administration (NOAA https://origin.cpc.ncep.noaa.gov/products/analysis_monitoring/ensostuff/ONI_v5.php) . Sendo 2009 um ano com baixa frequência de linhas de instabilidade, marcado pela ocorrência de El Niño e 2013 um ano com alta frequência de LI, apresentando-se neutro no que diz respeito ao ENOS.

Para a manipulação dos dados, utilizou-se o software Climate Date Operators (CDO), onde as médias anuais foram calculadas, e o software Grid Analysis and Display System (GrADS), onde as figuras dos campos médios espaciais de diferença foram geradas.

\section{RESULTADOS E DISCUSSÃO}

Nesta sessão são discutidos os principais resultados das características de grande escala associadas às diferenças das médias anuais de umidade específica dos dias com LI (cLI) e dos dias sem LI (sLI), verificando assim de que forma tais características podem estar relacionadas.

De acordo com a análise subjetiva das imagens de satélite, baseada na metodologia proposta por Cohen (1989), o ano de 2009 apresenta pouca frequência de LI, com 70 casos totais, sendo 44 LIC, 19 LIP1 e 7 LIP2. A configuração de grande escala presente neste ano é influenciada por um evento de El Niño, que ocasiona diminuição da precipitação na porção sul e oeste da bacia amazônica (MARENGO et al., 2011). 
A análise dos campos médios da diferença de umidade específica para o ano de 2009 (Figura 2) mostra áreas de diferenças positivas (acima de 0,2 $\mathrm{g} / \mathrm{Kg}$ ) em quase toda a extensão da América do Sul, exceto sobre os estados do Paraná e Santa Catarina, com valores inferiores a - $0,2 \mathrm{~g} / \mathrm{Kg}$ e sobre parte do Amazonas. Sobre a parte costeira das regiões Norte e Nordeste do Brasil observam-se as maiores diferenças positivas, com valores superiores a $0,4 \mathrm{~g} / \mathrm{Kg}$, sugerindo que o teor de umidade presente nos dias $\mathrm{CLI}$ é superior ao teor de umidade dos dias SLI. A análise dos vetores do vento (diferença), ainda para a Figura 2, sugere que o escoamento meridional é mais intenso, entretanto, apresenta deslocamento em direção ao oceano Atlântico Tropical ao atingir a costa N-NE do Brasil. Não é possível verificar a atuação da Alta Subtropical do Atlântico Sul (ASAS) no alcance da imagem gerada. Logo, sugere-se que a ASAS esteja mais deslocada para leste.

O ano de 2013 apresenta neutralidade quanto aos eventos de ENOS. Neste ano, verifica-se uma alta frequência de LI amazônicas, com um total de 198 casos, sendo 82 LIC, 73 LIP1 e 43 LIP2. Nos campos espaciais da diferença de umidade específica (Figura 3 ) observam-se as maiores diferenças positivas concentradas sobre a costa $\mathrm{N}-\mathrm{NE}$ do Brasil, onde as LI amazônicas se formam, com valores entre 0,2 e 0,8 g/Kg, que podem estar associados à atuação da Zona de Convergência Intertropical (ZCIT). Esses resultados sugerem ainda, que na média dos dias CLI há maior teor de umidade disponível na costa norte, favorecendo a formação e propagação das LI (ZHANG E KLEIN, 2010; OLIVEIRA E OYAMA, 2015). 
Figura 2: Campos médios da diferença de umidade específica $(\mathrm{g} / \mathrm{Kg})$ entre os dias $\mathrm{CLi}$ e sLI para o ano de 2009.

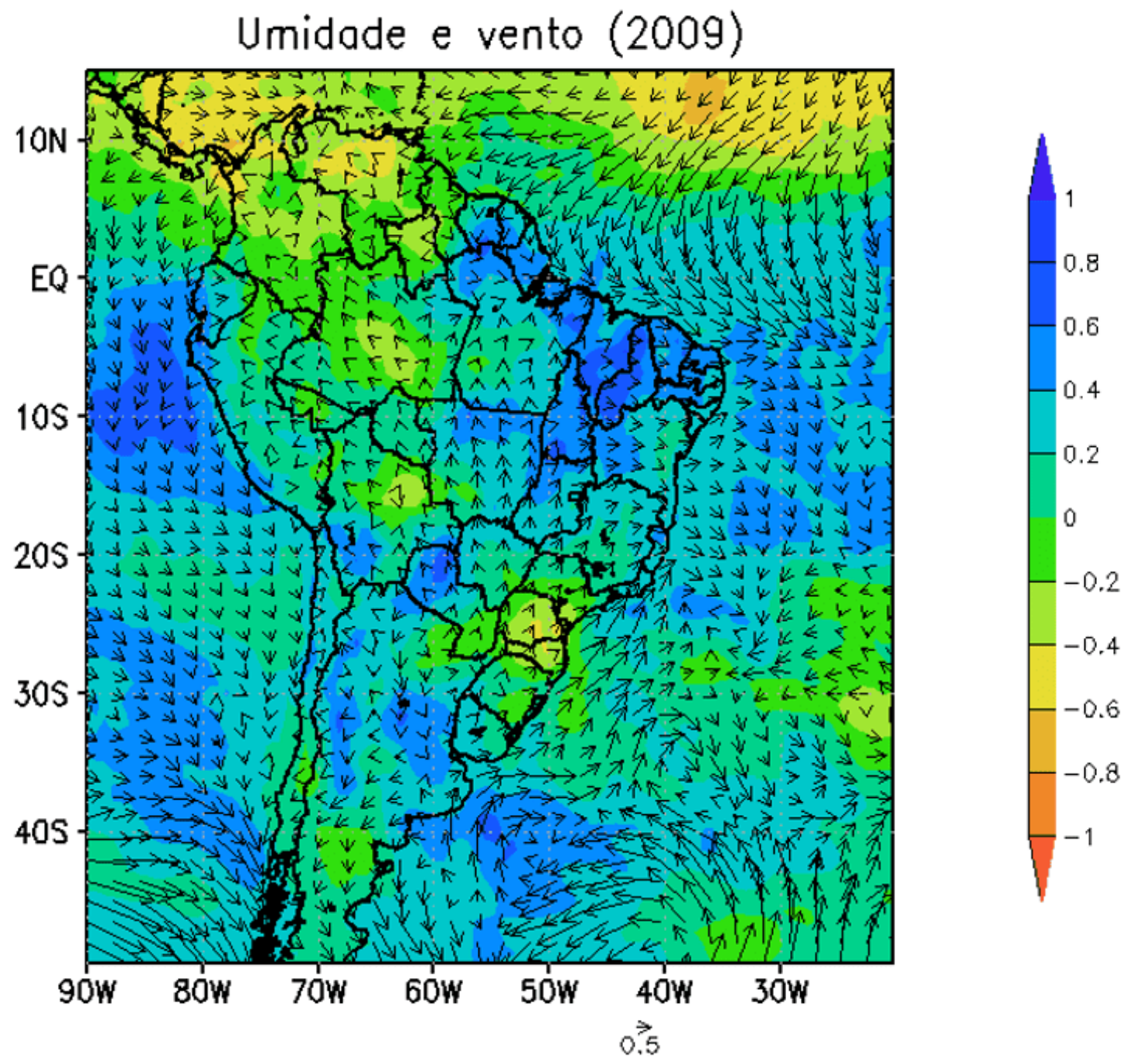

Fonte: Elaborada pelas autoras. 
Figura 3: Campos médios da diferença de umidade específica $(\mathrm{g} / \mathrm{Kg})$ entre os dias cLI e sLI para o ano de 2013.

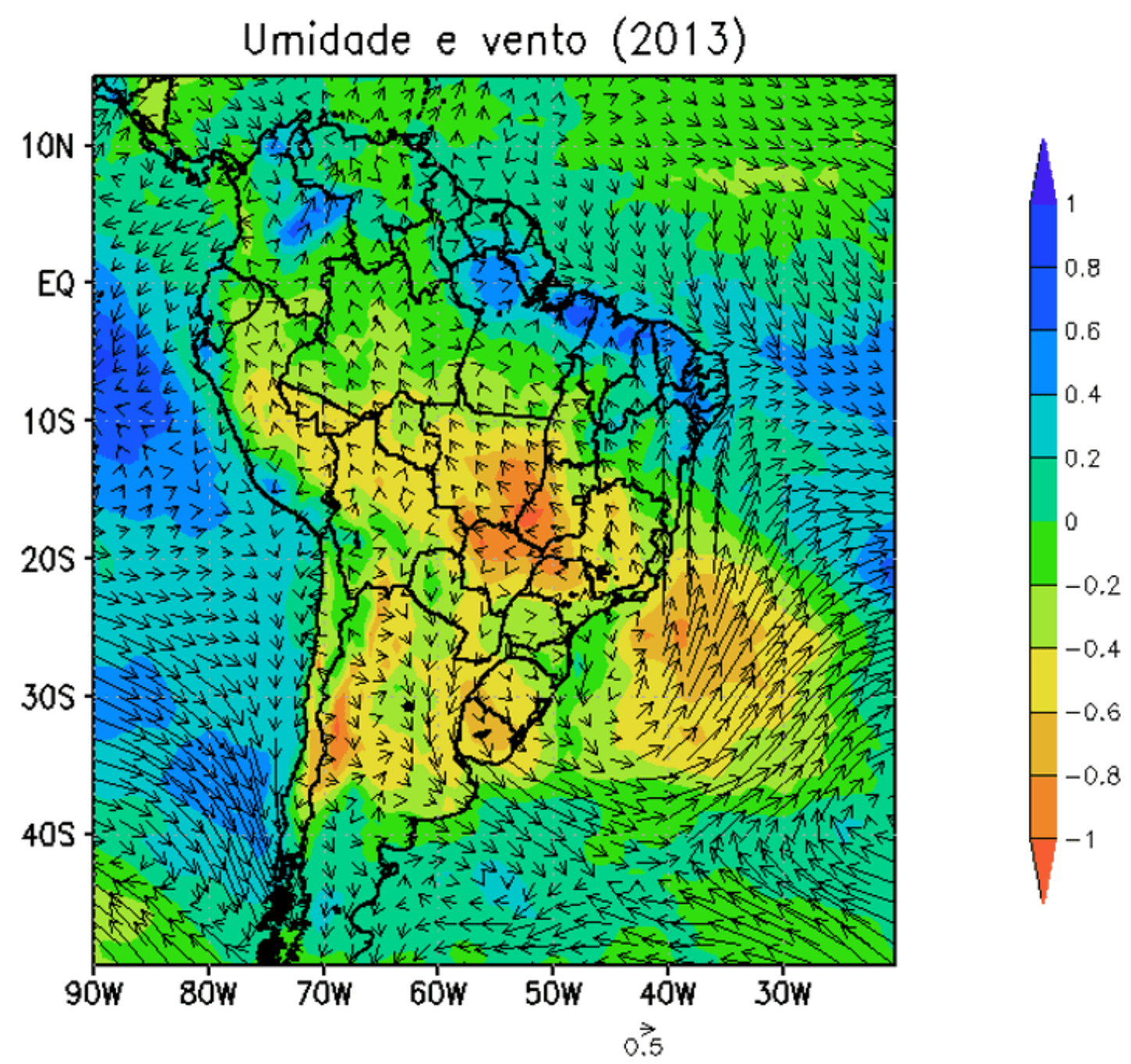

Fonte: Elaborada pelas autoras.

Além disso, é observado um núcleo relativamente seco sobre os estados do Mato Grosso e Goiás, com valores inferiores a $-1,0 \mathrm{~g} / \mathrm{Kg}$. Essa configuração de áreas com maior teor de umidade sobre a costa norte e nordeste brasileira e áreas com menor teor sobre a parte central do Brasil e sobre a região Sul e Sudeste, aparentam uma configuração de dipolo. Oliveira e Oyama (2015) indicam que a presença de um padrão de umidade semelhante a este age como uma célula de circulação direta, associada às anomalias de divergência e convergência, o que favorece a iniciação de LI, mas não a propagação do sistema. Logo, sugere-se que essa configuração de 
dipolo auxilia na formação e número de LI formadas no ano de 2013, mas não explica a quantidade de linhas propagantes identificadas neste ano.

Nos campos da diferença entre a média dos dias CLI e sLI (Figura 3) é observado na área de atuação da ASAS (aproximadamente centrada na porção sudeste do oceano Atlântico Sul) que há uma componente de sul mais intensa na média dos dias cLI, indicando que a ASAS se encontra mais intensa nos dias CLI. Sobre a faixa equatorial as componentes de norte são mais pronunciadas, podendo estar associadas com a Alta Subtropical do Atlântico Norte (ASAN). Com isto, verifica-se que na costa norte há um escoamento convergente, associado a ZCIT. Gloor et al. (2013) mostra que o transporte atmosférico de vapor d'água do Atlântico para a região amazônica aumenta cerca de $10 \%$ a precipitação no noroeste da Amazônia, confirmando então a importância do escoamento do vento sobre o aporte de umidade nas regiões costeiras.

Desta forma, as configurações de grande escala presentes no ano de 2009, com alto teor de umidade presente em quase toda a América do Sul nos dias CLI, a "ausência" relativa da atuação da ASAS sobre o litoral leste do $\mathrm{NE}$, agem inibindo o desenvolvimento de LI amazônicas. Enquanto no ano de 2013, a presença do padrão de dipolo associado à convergência na região equatorial sugere favorecimento do desenvolvimento e, possivelmente, da propagação das LI amazônicas.

\section{CONCLUSÃO}

Os resultados da análise dos campos médios anuais de diferença de umidade específica e vento entre os dias com LI e sem LI para os anos de 2009 (Figura 2) e 2013 (Figura 3) confirmam a relevância da variável umidade específica para a formação e propagação das linhas de instabilidade amazônicas, tendo em vista que nas duas figuras a região costeira, onde as LI se formam, permanece com diferenças positivas, o que significa que na média anual, os dias com linhas de instabilidade apresentam maior concentração de umidade tanto no ano de 2009, com menor frequência de LI e atuação de EI Niño, quanto no ano de 2013, com maior frequência de LI e neutralidade para o ENOS. Embora as duas figuras apresentem diferenças 
positivas, o ano de 2013 (Figura 3) é o que apresenta essas diferenças mais concentradas sobre a costa norte e nordeste do Brasil, enquanto em 2009, as diferenças de umidade na média anual revelam diferenças positivas mais distribuídas por boa parte do continente e costa oceânica próxima (Figura 2). Tal configuração permite supor que as diferenças positivas de umidade confinadas na região costeira podem ser indicativas de maior número de linhas de instabilidade formadas e propagadas, em oposição ao ano de 2009, que apesar de ter diferenças positivas mais espalhadas pelo continente, é um ano com baixa frequência de LI. Parece provável também que a neutralidade para ENOS em 2013 permite essa diferença positiva concentrada na costa norte e nordeste, enquanto as áreas ao sul desta região apresentam pouca diferença ou diferença negativa.

Com relação à diferença na intensidade e direção do vento, a Figura 2 (ano 2009) apresenta escoamento meridional mais forte em direção à costa, entretanto sem penetração no continente, havendo um desvio em direção ao oceano Atlântico Tropical, sugerindo que próximo à costa não há, na média anual de dias com LI, ventos tão mais fortes que na média anual de dias sem LI, criando assim um ambiente menos propício para a formação das linhas de instabilidade, com provável circulação de brisa menos intensa. A Figura 3 (ano 2013, com neutralidade em relação ao ENOS) apresenta baixa diferença de intensidade do vento em direção à costa norte do Brasil, mas ainda assim há predomínio do vento meridional em direção à costa, sugerindo que a umidade possa ser fator preponderante em relação à intensidade e direção do vento em 2013 (Figura 3) para o maior número de LI nesse ano, embora o escoamento do vento em direção à costa seja extremamente necessário para concentrar umidade sobre as regiões costeiras.

Vale destacar o padrão de dipolo em associação à convergência equatorial podendo ser um fator de favorecimento ao desenvolvimento e propagação das LI amazônicas no ano de 2013 (Figura 3). Enquanto no ano de 2009 (Figura 2), uma possível baixa intensidade dos ventos gerados pela circulação da ASAS penetrando o litoral leste do Nordeste nos dias CLI, pode agir para não favorecer o desenvolvimento de linhas de instabilidade. 
De acordo com os resultados discutidos acima, nota-se a complexidade da formação das linhas de instabilidade, que depende do comportamento de variadas condições ambientais para seu desenvolvimento e intensidade. Esse sistema contribui de forma expressiva para os volumes de precipitação não apenas no litoral norte e parte do litoral nordeste do Brasil, mas em boa parte da região Norte, pois não poucas vezes, algumas linhas de instabilidade propagam-se para o interior. É importante lembrar que a formação das LI pode sofrer influência do comportamento de várias variáveis, atuando ao mesmo tempo, bem como de configurações de grande escala. Estudar cada vez mais essas interações podem permitir maior compreensão sobre a formação do sistema e sua evolução, não apenas diária, mas a prazos maiores, sugerindo assim a continuação das pesquisas a respeito das linhas de instabilidade e de outras variáveis envolvidas na formação e propagação do sistema em razão da sua relevância.

\section{REFERÊNCIAS}

ALCÂNTARA, Clênia Rodrigues. Um estudo da relação entre jatos de baixos níveis e linhas de instabilidade da Amazônia. 2010. Tese de Doutorado. Universidade de São Paulo.

CAVALCANTI, Iracema Fonseca de Albuquerque. Um estudo sobre interações entre sistemas de circulação de escala sinótica e circulações locais. 1982. 133 p. (INPE-2494-TDL/097) Dissertação (Mestrado em Meteorologia) - Instituto Nacional de Pesquisas Espaciais, São José dos Campos, 1982.

CAVALCANTI, Iracema Fonseca de Albuquerque; FERREIRA, Nelson Jesus; SILVA DIAS, Maria Assunção Faus; JUSTI, Maria Gertrudes Alvarez. Tempo e clima no Brasil. São Paulo: Oficina de Textos, 2009, 463 p.

COHEN, Júlia Clarinda Paiva; SILVA DIAS, Maria Assunção Faus; NOBRE, Carlos Afonso. Aspectos climatológicos das linhas de instabilidade na Amazônia (Climatological features of squall lines over Amazonia). Climanálise, v. 4, n. 11, p. 3440, 1989. 
COHEN, Júlia Clarinda Paiva; SILVA DIAS, Maria Assunção Faus; NOBRE, Carlos Afonso. Environmental conditions associated with amazonian squall lines: a case study. Monthly Weather Review, v. 123, n. 11, p. 3163-3174, 1995.

GARSTANG, Michael; MASSIE, Harold L.; HALVERSON, Jeffrey; GRECO, Steven; SCALA, John. Amazon Coastal Squall Line. Part I: Structure and Kinematics. Monthly Weather Review, v. 122, n. 4, p. 608-622, 1994.

MARENGO, J. A., TOMASELLA, J., ALVES, L. M., SOARES, W. R., \& RODRIGUEZ, D. A. (2011). The drought of 2010 in the context of historical droughts in the Amazon region. Geophysical Research Letters, 38(12).

MOLION, Luiz Carlos Baldicero. On the dynamic climatology of the Amazon Basin and associated rain-producing mechanisms. The Geophysiology of Amazonia: Vegetation and Climate Interactions, Wiley Interscience, p. 391-407, 1987.

NEGRI, Andrew J.; ANAGNOSTOU, Emmanouil N.; ADLER, Robert. F. A 10-yr climatology of Amazonian rainfall derived from passive microwave satellite observations. Journal of Applied Meteorology, v. 39, n. 1, p. 42-56, 2000.

PEREIRA DE OLIVEIRA, Fernando; OYAMA, Marcos Daisuke. Antecedent atmospheric conditions related to squall-line initiation over the northern coast of Brazil in July. Weather and Forecasting, v. 30, n. 5, p. 1254-1264, 2015.

WIKIPÉDIA. Pará: Localização do Estado do Pará. Disponível em <https://pt.m.wikipedia.org/wiki/Par\%C3\%A1>. Acesso em: 30 jul. 2020.

WIKIPÉDIA. Belém (Pará): Localização de Belém do Pará. Disponível em $<$ https://pt.wikipedia.org/wiki/Bel\%C3\%A9m_(Par\%C3\%A1)>. Acesso em: 30 jul. 2020.

ZHANG, Yunyan; KLEIN, Stephen A. Mechanisms affecting the transition from shallow to deep convection over land: Inferences from observations of the diurnal cycle 
collected at the ARM Southern Great Plains site. Journal of the Atmospheric Sciences, v. 67, n. 9, p. 2943-2959, 2010.

Enviado: Setembro, 2020.

Aprovado: Setembro, 2020. 